\title{
A REVIEW OF LIMBAL STEM CELL DEFICIENCY
}

John E. Conto, OD, FAAO

Assistant Professor of Ophthalmology and Visual Science. Medical College of Wisconsin, Milwaukee, WI

Corresponding Author: jconto@mcw.edu

Submitted: February 24, 2019. Accepted: April 12, 2019. Published: June 10, 2019.

\begin{abstract}
Limbal stem cell deficiency (LSCD) can be secondary to multiple etiologies including contact lens wear, chemical or thermal trauma, and systemic disease, any of which can result in the reduction of the number of stem cells or their decreased functionality. Primary LSCD is seen with a variety of congenital anterior segment disorders. Often LSCD can be stabilized and timely diagnosis is the key. The use of topical corticosteroids and artificial tear lubricants, along with treatment of any underlying conditions, and discontinuation of contact lenses are important initial treatment strategies. Advance treatments include amniotic membranes, limbal stem cell transplantation and keratoprostheses.
\end{abstract}

Key Words: limbal stem cell deficiency, corticosteroids, hydrogel contact lenses

Davanger and Evensen discussed the possibility that the pericorneal papillary structure may be the generator of corneal epithelium in $1971 .{ }^{1}$ Subsequent studies demonstrated that limbal stems cells (LSC) were found to be long-lived, self-renewing, and played a vital role in the regeneration of corneal epithelial cells. ${ }^{2-5}$ The corneal LSCs arise from the peripheral epithelial basal layer and also act as a junctional barrier to the ingress of conjunctival cells onto the corneal surface. ${ }^{6}$ Damage to the LSC can result in either a focal or wide spread conjunctival invasion of the cornea, with complications of a dysfunctional tear film, persistent epithelial defects, pannus and neovascularization of the cornea. Opacification, melting and perforation of the cornea is a possibility in severe cases. ${ }^{7}$

Patients with LSC deficiency (LSCD) can be asymptomatic but likely present with a variety of symptoms including decreased vision, tearing and ocular discomfort or pain. LSCD can result from external causes, including chemical or thermal injury, chemotherapy, Steven-Johnson syndrome, ocular surface disease, and the abuse of hydrogel contact lenses. ${ }^{8}$ Etiologies of primary LSCD include aniridia, epidermal dysplasia and Turner syndrome. Iatrogenic causes from ocular surgery can also cause LSCD, especially when multiple surgeries have been performed. ${ }^{9,10}$

Diagnosis of LSCD can be challenging due to the non-specific symptoms and signs at presentation. There is not one classic symptom that is indicative of LSCD, and biomicroscopy findings are crucial in making the diagnosis. As the ocular surface manifestations can be quite variable and dependent on the extent of limbal involvement, conjunctivalization of the corneal surface is the most reliable sign. ${ }^{11}$ Ziadi, et al., described LSCD as a "clinical chameleon." 12 The use of vital stains can assist in the diagnosis as well as imaging techniques and histologic tests. Treatment is directed towards reducing the inflammatory response and restoring the stem cells. The condition can present as partial or total and be progressive. The use of amniotic membranes, scleral lenses and limbal cell transplantation has increased the success of stabilizing LSCD. ${ }^{13}$ 


\section{OVERVIEW}

In 2000, Dua, et al, reviewed LSCD. ${ }^{11}$ They characterized the limbal basal epithelium being the least differentiated, and lacking the keratin phenotype markers seen in other corneal cell populations. The peripheral basal cells are also highly proliferative and respond to central corneal injuries. This exclusive location for the corneal epithelial stem cells was also supported by the observation of abnormal corneal wound healing when the limbal tissue was disturbed. ${ }^{14,15}$ The location of stem cells in the limbal region allows for protection against solar damage (highly pigmented) and makes the cells less susceptible to shearing forces from trauma and other environmental forces. ${ }^{16}$

The pathology of LSCD arises from either destructive loss of the LSC or the dysfunction of the limbal niche. Primary LSCD arises from conditions that do not support stem cell functions such as seen in aniridia, Peter's anomaly and other congenital disorders. Most cases of LSCD are secondary and due to external causes as described prior. ${ }^{17}$ LSCD may be focal or diffuse and stationary or progressive. LSCD also has been described in the literature as partial and total. There does not appear to be a widely agreed upon designation. Jawaheer, et al, suggested the following in their review of the literature ${ }^{7}$ (Table 1):

A recent paper by Vazirani, et al, reviewed the demographic features and causes of with LSCD in 1331 patients. ${ }^{18}$ They reported that unilateral was more common than bilateral ( $59.4 \%$ versus $40.6 \%$ ). Males comprised $65.7 \%$ and the median age was 24 -years. Total LSCD accounted for $67 \%$ of eyes affected.
The most common cause of unilateral LSCD was ocular surface burns (83.73\%). In bilateral cases, the causes were more varied, with ocular surface burns (29.95\%), allergic conjunctivitis (29.48\%), StevensJohnson syndrome (23.11\%), aniridia (9.43\%), and mucous membrane pemphigoid (3.54\%). LSCD from contact lenses was not a significant cause; however, this study was performed in India and may reflect a patient population bias.

Often, LSCD has often been described as focal when secondary to contact lenses. Reviews indicate that $15-73 \%$ cases of focal LSCD are due to CLs. ${ }^{19,20}$ Some have suggested that the term "limbal stem cell dysfunction" would be more appropriate in these cases. ${ }^{21}$ The clinical signs are typically limited to the superior cornea with projections of whorl-like epithelial opacities. The focal nature of the LSCD is thought to be caused by a disruption of the limbal cell environs rather than a loss of LSC. ${ }^{22,23}$ Contact lensrelated LSCD appears to be from a combination of mechanical, hypoxic and toxic effects. In the past, the use of thimerosal-based solutions has been linked. ${ }^{24}$

Termote and associates recently reported on LSCD in contact lens patients. ${ }^{25}$ In the study population, LCSD occurred in about $3 \%$ of contact lens wearers. All the patients were hydrogel contact lens wearers. Key elements of this study related to contact lens wear included:

1. Mean age was about 38 years. $71 \%$ females, no racial preference;

2. Average contact lens wearing time of 18 years, 11 hours a day;

TABLE 1 Types of LSCD

\begin{tabular}{|l|l|}
\hline \multicolumn{1}{|c|}{ Total LSCD } & \multicolumn{1}{c|}{ Partial LSCD } \\
\hline 4 quadrants neovascularization & $2-3$ quadrants of neovascularization \\
\hline circular conjunctivalization of entire limbus & $120^{\circ}$ to less than $360^{\circ}$ of limbal involvement \\
\hline complete disappearance of the palisades of Vogt & diffuse NaFL with/without epithelial defects \\
\hline 360 degrees of conjunctivalization & partial conjunctivalization of the cornea \\
\hline Complete loss or unable to detect LSC & \\
\hline
\end{tabular}

Adapted from Jawaheer, et al. ${ }^{7}$

LSCD = limbal stem cell deficiency

J Dry Eye Ocul Sur Dis 2(1):e4-e11; June 10, 2019

This article is distributed under the terms of the Creative Commons Attribution-Non Commercial 4.0 International License. (C2019 Conto. 
3. All cases were superior and $60 \%$ were bilateral;

4. $71 \%$ reported wearing silicone hydrogel lenses and $53 \%$ wore them longer than the manufacture's recommendation;

5. $71 \%$ monthly replacement and $29 \%$ bi-weekly disposable lens;

There were numerous brands of contact lenses as well as disinfection solutions used by the subjects, and the study results seemed to reflect more of the popularity of the products than a relational effect. Treatment included topical corticosteroids and preservative-free artificial tears. Average use of the steroid drops was about 8.5 months. Fifty nine percent of patients returned to contact lens wear about 6.5 months after diagnosis. Patients typically switched to daily disposable lenses and reduced their wearing time. Most continued to use the same disinfection solution/method as before.

Patients (59\%) demonstrated some type of ocular surface disease, mainly dry eye. Blepharitis was also seen, but less than dry eye disease. Visual acuity improved in nearly $75 \%$ of the patients from presentation, and none of the patients needed surgery to control the LSCD.

Mechanical trauma may play a more significant role in contact lens-related LSCD. Lenses are designed to move and inadequate movement may lead to dehydration and increased friction to the ocular surface. ${ }^{26,27}$ The interaction between lid blinking and contact lens movement impact the superior limbus particularly and likely contributes to why LSCD in contact lens patients more frequently affects the superior cornea. ${ }^{28,29}$

Because of the variability of ingredients in disinfecting solutions, it is difficult to make any conclusions of their relationship to LSCD. The limbus is particularly sensitive to chemical insult and adverse toxic effects from solutions, along with the mechanical trauma, are likely to contribute to LSCD in susceptible patients. The impact of mini-scleral and scleral lenses has not been extensively studied either from a causative or therapeutic perspective.

\section{DIAGNOSIS}

Diagnosis of LSCD can encompass examination for the clinical signs, impression cytology to identify conjunctival goblet cells, spectral-domain optical coherence tomography to detect corneal thinning and confocal microscopy to discover alterations in the limbal stromal niche. Identification of LSCD by the use of cytoplasmic or nuclear markers has been difficult. A genetic marker, $\mathrm{ABCB} 5$, has been proposed to identify limbal stem cells. ${ }^{30}$ This marker appears to be involved in the maintenance of the LSC.

The clinical features seen in LSCD are most evident with careful slit-lamp biomicroscopy examination. While there is no clear and consistent grading system for LSCD, Le, et al, did described staging related to the ocular surface findings (17) (Table 2)(Figures 1-3).

Impression cell cytology has facilitated the diagnosis of LSCD and helps to differentiate LSCD from other

TABLE 2 Grades and Stages of LSCD

\begin{tabular}{|c|c|}
\hline Stage & Characteristics \\
\hline Mild stage (Grade 1) & $\begin{array}{l}\text { - reduction in the corneal reflex from irregular surface and peripheral corneal } \\
\text { opacification or conjunctivalization of the epithelium } \\
\text { - fluorescein pooling with late staining or stippling } \\
\text { - thinning or loss of palisades of Vogt, flattening of the limbus }{ }^{8}\end{array}$ \\
\hline Moderate stage (Grade 2) & $\begin{array}{l}\text { - grade } 1 \text { findings } \\
\text { - displays vortex keratopathy, superficial vascularization or peripheral pannus } \\
\text { - migrated conjunctival epithelial cells attract new blood vessel formation }{ }^{31}\end{array}$ \\
\hline Severe stage (Grade 3) & $\begin{array}{l}\text { - } \text { grade } 1 \text { and } 2 \text { findings } \\
\text { - } \text { persistent epithelial defects } \\
\text { - } \text { stromal neovascularization } \\
\text { - scarring and opacification }\end{array}$ \\
\hline
\end{tabular}

Adapted from Le, et al. ${ }^{8,17}$ and Dua. ${ }^{31}$

J Dry Eye Ocul Sur Dis 2(1):e4-e11; June 10, 2019

This article is distributed under the terms of the Creative Commons Attribution-Non

Commercial 4.0 International License. (C2019 Conto. 
FIG. 1 Partial (Grade 1) LSCD secondary to hydrogel contact lens, with conjunctival extension onto superior cornea.

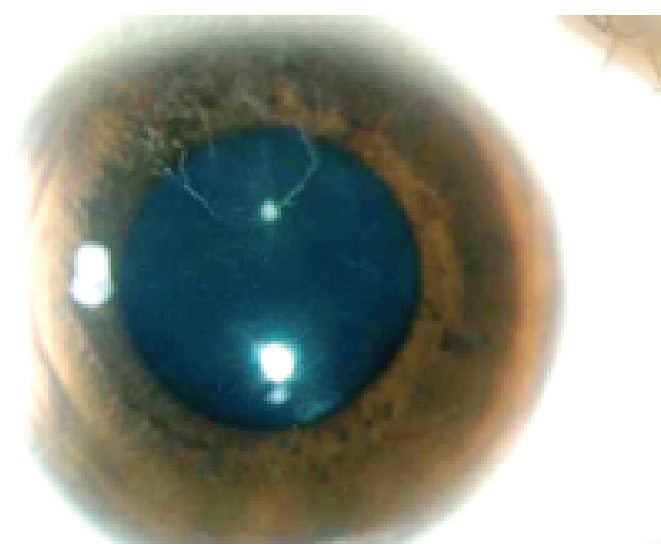

FIG. 2 Partial (Grade 2) LSCD secondary to chemical exposure, with conjunctivalization greater than $2 / 3$ of cornea, pannus and scarring of inferior cornea.

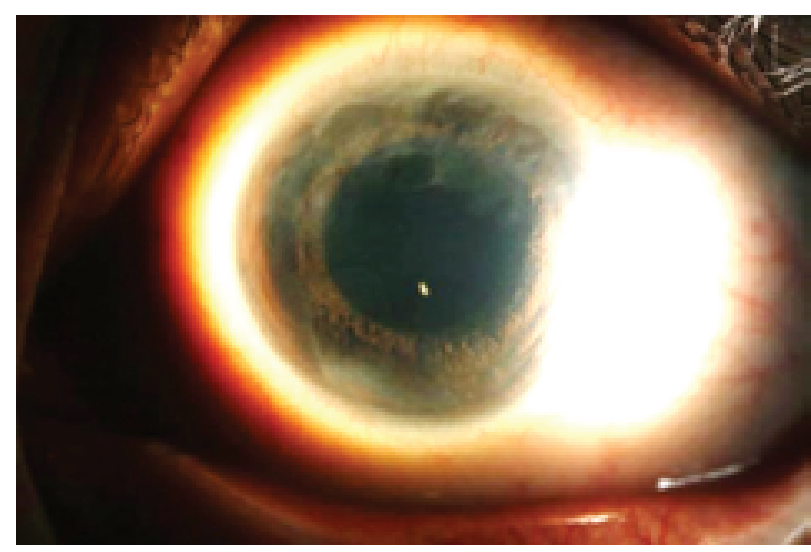

ocular surface disease conditions, including vitamin A deficiency, squamous metaplasia and psoriasis. ${ }^{32}$ It can be implemented in the out-patient setting, although it does require resulting by either a histologist or an ocular pathologist, which has limited the utilization.

The goal of impression cytology is to detect conjunctival goblet cells rather than identifying limbal stem cells. It is also a useful adjunct in distinguishing variants of dry eye, particular mucin deficiency. ${ }^{33}$ This technique preserves the limbal stem cells without reducing the population further as seen in surgical biopsies of the limbus. The presence of goblet cells on the surface of the cornea is a definitive diagnosis
FIG. 3 Partial (Grade 3) LSCD secondary mucous membrane pemphigoid, with central conjunctivalization, corneal hypertrophy and hemosiderosis.

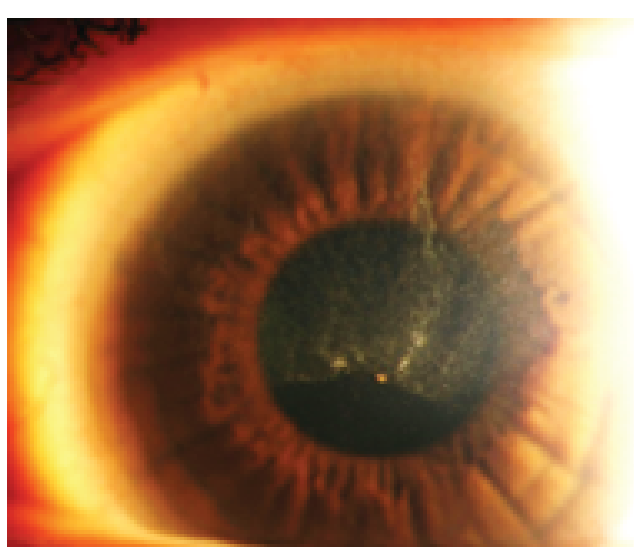

of conjunctival ingrowth; however, it may not detect early changes. Repeated testing is suggested. Advance cases of LSCD may not show goblet cells presence due to keratinization of the cornea, producing a false negative. A study of impression cytology in total LSCD patients, showed that $100 \%$ of subjects had goblet cells on samples. ${ }^{34}$ It also demonstrated the presence of squamous metaplasia in $75 \%$ and inflammatory cells in $70 \%$ of cases. Another study by Sacchetti, and associates, confirmed goblet cell detection by impression cytology verified the diagnosis of LSCD, but that $18 \%$ of patients referred for LSCD showed a normal corneal cell phenotype rather than conjunctival epithelium. ${ }^{35}$ They suggested that the presence of cytokeratin-19, a structural protein seen in the conjunctiva but not in the cornea, may be a better marker in detecting conjunctival epithelium on the cornea. Cytokeratin-19 was found in $82 \%$ of samples, while goblet cells were found in only $59 \%$. The report also indicated a high correlation between decreased corneal sensation and these cytological findings.

The use of anterior segment spectral-domain optical coherence tomography (ASOCT) has gained popularity as a valued instrument to assist in the diagnosis of LSCD. ASCOT is efficient and accurate, with low risk since it is a noncontact method. ${ }^{36}$ The corneal epithelial layer can be visualized and the thickness measured. Patients with LSCD will show a greater difference between the minimal and maximal epithelial thickness. In addition, the limbal region and palisades 
of Vogt (POV) can be identified and evaluated in vivo. ${ }^{37}$ The transition zone between the hyporeflective corneal epithelium and hyperreflective conjunctival epithelium can be distinguished with ASOCT.

The most common limbal ASOCT characteristics seen in patients with LSCD include flattening of the stroma and thinner epithelium (cross sections parallel) and a complete flat profile to the limbal transition zone (cross sections perpendicular). There is also absence of POV and limbal crypts that are typically visualized in normal eyes. Loss of the basement membrane (84\%) and of Bowman layer (79\%) was seen in LSCD. Subepithelial fibrosis was present in $90 \%$ of patients. These findings were relatively equal in distribution for all regions. ${ }^{38}$

While less frequently used, in vivo confocal microscopy (IVCM) is an invaluable procedure to aid in the diagnosis of LSCD. It can perform live imaging of the corneal and limbal epithelium. Major drawbacks include cost of the instrument and a trained technician; it is invasive with direct contact of the cornea; and the field of view is limited. It also requires an adept provider to interpret the images.

Chan, et al, concluded by using IVCM, that the peripheral and central corneal thickness is decreased in LSCD. ${ }^{39}$ Corneal thickness was reduced by $20 \%$ centrally and 39\% at the limbus as compared to controls. Others have reported that there was no variance between mean central corneal thickness in LSCD and normal patients; however, the maximal to minimal difference in corneal thickness was greater in LSCD. ${ }^{38}$

The limbal structural changes between total, partial and focal LSCD are variable and can be imaged by IVCM. The cell phenotype in total LSCD was conjunctival, and mixed in partial. Conjunctival ingrowth, superficial and deep vascularization, as well as, the presence of inflammatory cells can be detected with IVCM. Inflammatory cells can be present even in non-affected areas in partial..$^{40}$ This is an important prognostic factor in transplantation outcome. ${ }^{41}$ The subbasal nerves are thinned and sparsely distributed in focal or partial, while absent in total LSCD. This is a critical factor in corneal sensitivity and the development of neurotrophic keratitis.

Araújo and associates report that IVCM can also confirm LSCD $76 \%$ of time, by visualization of the conjunctival epithelial ingrowth on the corneal surface. ${ }^{34}$ Other findings include goblet cells, inflammatory cells, corneal neovascularization and diffuse hyperreflection of the stroma.

In summary, most cases of LSCD are diagnosed by careful clinical examination. At this time, other methods are typically used to support the diagnosis and assist in therapeutic strategies.

\section{MANAGEMENT}

The management of LSCD is varied and dependent on the clinical appearance, whether it is unilateral or bilateral and partial or total. There has been significance progress in the treatment of LSCD over the past decade utilizing both medical and surgical approaches. The first line of therapy is always to address the inflammatory response and remove the underlying cause if possible. Initial medical management is aimed at the restoring the homeostasis of the limbal zone. In total LSCD, medical management is often not effective and requires surgical intervention.

In partial or focal LSCD, management of pulsing with variable dosing of topical corticosteroids such as prednisolone acetate $1 \%$ or loteprednol etabonate $0.5 \%$ is considered to be first-line therapy for most cases. ${ }^{21}$ The use of compounded preservative-free methylprednisolone $1 \%$ is also an option. There are limited references on the use of either fluorometholone $0.1 \%$ or difluprednate $0.05 \%$, and use of NSAID agents has not been discussed. The treatment of underlying ocular surface disease and discontinuation of contact lens wear is a key factor. Often LSCD patients have poor tear dysfunction and persistent intensive lubrication of the ocular surface is critical. This underscores the importance of treating ocular surface disease, including rosacea, prior to the fitting of hydrogel lenses to reduce the possibility of focal LSCD. When more than 4 clock hours of the limbus is involved, LSCD is likely to persist greater than 3 months. ${ }^{21}$ Patients may need to be on corticosteroids for longer periods and early withdrawal can lead to under treatment. At least a month of corticosteroid use produced a significant clinical response in a study by Kim, et al. ${ }^{21}$ In this series, corticosteroids were tapered off in $82 \%$ of patient in 2-3 months. Most patients that respond to conservative medical management

J Dry Eye Ocul Sur Dis 2(1):e4-e11; June 10, 2019

This article is distributed under the terms of the Creative Commons Attribution-Non

Commercial 4.0 International License. (C2019 Conto. 
may require $12-15$ months of treatment to stabilize the ocular surface signs.

After initial treatment with corticosteroids, cyclosporine $0.5 \%$ can be used to discourage the inflammatory response. Lifitegrast $5 \%$ may be a possible alternative to cyclosporine. ${ }^{42}$ Compounded $0.03 \%$ tacrolimus ointment or drops have been shown to control underlying inflammation from ocular surface disease. ${ }^{43}$ Other adjunctive therapeutics includes topical vitamin A ointment, punctal plug occlusion and autologous serum drops. Scleral lenses may prove to be a useful modality in partial LSCD. ${ }^{21,44}$

The use of amniotic membrane (AM) patches in the treatment of LSCD is well established. ${ }^{45,46}$ When used as a graft, AM is typically glued or sutured in place. Temporary AMs (cryopreserved such as PROKERA ${ }^{\circledR}$ or dehydrated such as AmbioDisk ${ }^{\mathrm{TM}}$ ) can also be used in cases that do not respond to conservative therapy. ${ }^{47,48}$ While not providing stem cells, these membranes support the regeneration of LSC. A surgical permanent graft is preferred in total chemical injuries, since the palpebral conjunctiva is often involved, and the AM can be extended onto the conjunctival surface. A recent paper by Baradaran and associates studied the use of amniotic membrane extracted eye drops (AMEED) to aid in the cultivation of LSCs. ${ }^{49}$

There are many surgical approaches that have been developed over the past 2 decades in the management of LSCD. ${ }^{13,50-52}$ All limbal transplantations provide either allogeneic or autologous cell sources for the graft. Partial LSCD can use autologous limbal tissue from the uninvolved eye. ${ }^{53,54} \mathrm{~A}$ concern for autologous transplantation is iatrogenic damage to the donor site. Ex vivo cultivation of a small sample from the autologous cells minimizes the harm and increases the success rates of LSC restoration. ${ }^{41,55}$

Bilateral LSCD presents with more challenges and poorer outcomes in visual rehabilitation. Since bilateral LSCD is more often from burn injuries or cicatricial condition, the accompanying damage to surrounding ocular tissue also complicates recovery. These issues must be appropriately addressed prior to ocular surface surgical intervention. Therapy is either cell-based procedures or keratoprostheses.

Cell-based procedures for bilateral LSCD include allogeneic limbal stem cell epithelial transplantation
(LSCT) and cultivated oral mucosal transplantation (COMET). Ex vivo cultivated LSCT has largely replaced direct methods ${ }^{56}$ While direct transplantation has had reasonable outcomes, disadvantages include delay of several weeks in the re-epithelization of the recipient surface and a large degree of donor tissue is needed. AMs are then used to transfer the cultivated cells to the host. The most concerning complication of allogeneic LSCT is rejection. For this reason, the use of postoperative systemic immunosuppressants is recommended. Baylis, et al, reported a long-term success rate for a stable corneal surface of $73 \% .^{57}$

COMET can provide alternative allogeneic sources since the oral mucosa shares certain traits with LSC. A small biopsy from the oral mucosa is extracted and then cultivated ex vivo. An AM is then seeded and then transplanted onto the recipient's corneal surface. ${ }^{58,59}$ Long-term outcomes have not been as successful as LSCT. ${ }^{60}$

Keratoprostheses can be used when there is substantial corneal scarring and high likelihood of corneal transplant failure. The procedure is similar to penetrating keratoplasty. The Boston keratoprostheses (KPro) is the most commonly used device and has been in continuous development by Dohlman and associates. ${ }^{61}$ There are several types, dependent if there is adequate wetting of the ocular surface. After the procedure, a large diameter hydrogel bandage contact lenses is placed indefinitely over the prosthesis with maintenance use of broad-spectrum antimicrobial agents. ${ }^{56}$ For patients with adequate tear function, the detention rate for 2 years for the Boston KPro Type 1 device has been reported to exceed $90 \%{ }^{62}$

The management for both partial and total LSCD has improved over the past several decades and promise of better therapies, including induced pluripotent stem cells (iPSCs) that can be transformed into limbal cells are on the horizon. ${ }^{63}$ The continued improvement of scleral lenses, such as EyePrintPRO ${ }^{\mathrm{TM}}$, BostonSight ${ }^{\circledR}$ PROSE or Zenlens ${ }^{\mathrm{TM}}$, also seem to show potential as an adjunct therapy. ${ }^{64}$

\section{CONCLUSION}

LSCD is caused by many etiologies, as in this case, secondary to hydrogel contact lenses. The overall goal of treatment is to reduce the inflammatory response 
and to stabilize the ocular surface environment. If conservative treatments are not successful then surgery may be necessary to rehabilitate the vision. This case report highlights the current diagnosis techniques and treatment strategies in LSCD.

\section{DISCLOSURE}

No financial interest in materials or products. No grant funding in support

\section{REFERENCES}

1. Davanger M, Evensen A. role of the pericorneal papillary structure in renewal of corneal epithelium. Nature 1971;229:560.

2. Thoft RA, Wiley LA, Sundarraj N. The multipotential cells of the limbus. Eye 1989;3:109.

3. Thoft RA, Friend J. Biochemical transformation of regenerating ocular surface epithelium. Invest Ophthalmol Vis Sci 1977;16(1):14-20.

4. Shapiro MS, Friend J, Thoft RA. Corneal re-epithelialization from the conjunctiva. Invest Ophthalmol Vis Sci 1981;21(1 Pt 1):135-42.

5. Sun TT, Lavker RM. Corneal epithelial stem cells: past, present, and future. The journal of investigative dermatology Symposium proceedings 2004;9(3):202-7.

6. Tseng SCG. Concept and application of limbal stem cells. Eye 1989;3:141.

7. Jawaheer L, Anijeet D, Ramaesh K. Diagnostic criteria for limbal stem cell deficiency-a systematic literature review. Surv Ophthalmol 2017;62(4):522-32.

8. Le Q, Yang Y, Deng SX, Xu J. Correlation between the existence of the palisades of Vogt and limbal epithelial thickness in limbal stem cell deficiency. Clin Exp Ophthalmol 2017;45(3):224-31.

9. Bobba S, Di Girolamo N, Mills R, et al. Nature and incidence of severe limbal stem cell deficiency in Australia and New Zealand. Clin Exp Ophthalmol 2017;45(2):174-81.

10. Schwartz GS, Holland EJ. Iatrogenic limbal stem cell deficiency. Cornea 1998;17(1):31-7.

11. Dua HS, Azuara-Blanco A. Limbal stem cells of the corneal epithelium. Surv Ophthalmol 2000;44(5):415-25.

12. Zaidi FH, Bloom PA, Corbett MC. Limbal stem cell deficiency: a clinical chameleon. Eye (Lond) 2003;17(7):837-9.

13. Holland EJ. Management of limbal stem cell deficiency: a historical perspective, past, present, and future. Cornea 2015;34 Suppl 10:S9-15.
14. Chen JJ, Tseng SC. Corneal epithelial wound healing in partial limbal deficiency. Invest Ophthalmol Vis Sci 1990;31(7):1301-14.

15. Huang AJ, Tseng SC. Corneal epithelial wound healing in the absence of limbal epithelium. Invest Ophthalmol Vis Sci 1991;32(1):96-105.

16. Hogan MJ AJ, Weddell JE. Histology of the human eye. 1971:112-26.

17. Le Q, Xu J, Deng SX. The diagnosis of limbal stem cell deficiency. Ocul Surf 2018;16(1):58-69.

18. Vazirani J, Nair D, Shanbhag S, Wurity S, Ranjan A, Sangwan V. Limbal stem cell deficiency-demography and underlying causes. Am J Ophthalmol 2018;188:99-103.

19. Donisi PM, Rama P, Fasolo A, Ponzin D. Analysis of limbal stem cell deficiency by corneal impression cytology. Cornea 2003;22(6):533-8.

20. Kim KH, Kim WS. Corneal limbal stem cell deficiency associated with the anticancer drug S-1. Optom Vis Sci 2015;92(4 Suppl 1):S10-3.

21. Kim BY, Riaz KM, Bakhtiari P, et al. Medically reversible limbal stem cell disease: clinical features and management strategies. Ophthalmology 2014;121(10):2053-8.

22. Martin R. Corneal conjunctivalisation in long-standing contact lens wearers. Clin Exp Optom 2007;90(1):26-30.

23. Jeng BH, Halfpenny CP, Meisler DM, Stock EL. Management of focal limbal stem cell deficiency associated with soft contact lens wear. Cornea 2011;30(1):18-23.

24. Nguyen DQ, Srinivasan S, Hiscott P, Kaye SB. Thimerosal-induced limbal stem cell failure: report of a case and review of the literature. Eye Contact Lens 2007;33(4):196-8.

25. Termote K, Schendel S, Moloney G, Holland SP, Lange AP. Focal limbal stem cell deficiency associated with soft contact lens wear. Can J Ophthalmol J. 2017;52(6):552-8.

26. Holden BA, Stephenson A, Stretton S, et al. Superior epithelial arcuate lesions with soft contact lens wear. Optom Vis Sci 2001;78(1):9-12.

27. Rossen J, Amram A, Milani B, et al. Contact lensinduced limbal stem cell deficiency. Ocular Surface 2016;14(4):419-34.

28. Holland EJ, Schwartz GS. Iatrogenic limbal stem cell deficiency. Trans Am Ophthalmol Soc 1997;95:95-107; discussion -10 .

29. Chan CC, Holland EJ. Severe limbal stem cell deficiency from contact lens wear: patient clinical features. Am J Ophthalmol 2013;155(3):544-9 e2. 
30. Ksander BR, Kolovou PE, Wilson BJ, et al. ABCB5 is a limbal stem cell gene required for corneal development and repair. Nature 2014;511(7509):353-7.

31. Dua HS. The conjunctiva in corneal epithelial wound healing. Br J Ophthalmol 1998;82(12):1407-11.

32. Kim KH, Mian SI. Diagnosis of corneal LSCD. Curr Opin Ophthalmol 2017;28(4):355-62.

33. Bron AJ, Mengher LS. The ocular surface in keratoconjunctivitis sicca. Eye (Lond). 1989;3 ( Pt 4):428-37.

34. Araujo AL, Ricardo JR, Sakai VN, et al. Impression cytology and in vivo confocal microscopy in corneas with total LSCD. Arq Bras Oftalmol 2013;76(5):305-8.

35. Sacchetti M, Lambiase A, Cortes M, et al. Clinical and cytological findings in limbal stem cell deficiency. Graefes Arch Clin Exp Ophthalmol 2005;243(9):870-6.

36. Lathrop KL, Gupta D, Kagemann L, Schuman JS, Sundarraj N. Optical coherence tomography as a rapid, accurate, noncontact method of visualizing the palisades of Vogt. Invest Ophthalmol Vis Sci 2012;53(3):1381-7.

37. Espandar L, Steele JF, Lathrop KL. Optical coherence tomography imaging of the palisades of Vogt to assist clinical evaluation and surgical planning in a case of limbal stem-cell deficiency. Eye Contact Lens 2017;43(5):e19-e21.

38. Banayan N, Georgeon C, Grieve K, Borderie VM. Spectral domain optical coherence tomography in limbal stem cell deficiency. A Case Control Study Am J Ophthalmol 2018.

39. Chan EH, Chen L, Yu F, Deng SX. Epithelial thinning in limbal stem cell deficiency. Am J Ophthalmol 2015;160(4):669-77 e4.

40. Chidambaranathan GP, Mathews S, Panigrahi AK, et al. In vivo confocal microscopic analysis of limbal stroma in patients with limbal stem cell deficiency. Cornea 2015;34(11):1478-86.

41. Pellegrini G, Rama P, Di Rocco A, et al. Concise review: hurdles in a successful example of limbal stem cell-based regenerative medicine. Stem Cells 2014;32(1):26-34.

42. Cheung AY, Sarnicola E, Govil A, Holland EJ. Combined conjunctival limbal autografts and living-related conjunctival limbal allografts for severe unilateral ocular surface failure. Cornea 2017;36(12):1570-5.

43. Shoughy SS, Jaroudi MO, Tabbara KF. Efficacy and safety of low-dose topical tacrolimus in vernal keratoconjunctivitis. Clin Ophthalmol (Auckland, NZ) 2016;10:643-7.
44. Chahal HS, Estrada M, Sindt CW, et al. Scleral contact lenses in an academic oculoplastics clinic: epidemiology and emerging considerations. Ophthal Plastic Reconstr Surg 2017.

45. Paolin A, Cogliati E, Trojan D, et al. Amniotic membranes in ophthalmology: long term data on transplantation outcomes. Cell Tissue Banking 2016;17(1):51-8.

46. Sabater AL, Perez VL. Amniotic membrane use for management of corneal limbal stem cell deficiency. Curr Opin Ophthalmol 2017;28(4):363-9.

47. Kheirkhah A, Johnson DA, Paranjpe DR, et al. Temporary sutureless amniotic membrane patch for acute alkaline burns. Arch Ophthalmol 2008;126(8): 1059-66.

48. Kolomeyer AM, Do BK, Tu Y, Chu DS. Placement of ProKera in the management of ocular manifestations of acute Stevens-Johnson syndrome in an outpatient. Eye Contact Lens 2013;39(3):e7-11.

49. Baradaran-Rafii A, Asl NS, Ebrahimi M, et al. The role of amniotic membrane extract eye drop (AMEED) in in vivo cultivation of limbal stem cells. Ocul Surf 2018;16(1):146-53.

50. Daya SM. Conjunctival-limbal autograft. Curr Opin Ophthalmol 2017;28(4):370-6.

51. He H, Yiu SC. Stem cell-based therapy for treating limbal stem cells deficiency: A review of different strategies. Saudi J Ophthalmol 2014;28(3):188-94.

52. Atallah MR, Palioura S, Perez VL, Amescua G. Limbal stem cell transplantation: current perspectives. Clin Ophthalmol (Auckland, NZ) 2016;10:593-602.

53. Kenyon KR. Limbal autograft transplantation for chemical and thermal burns. Dev Ophthalmol 1989;18:53-8.

54. Vazirani J, Basu S, Kenia H, et al. Unilateral partial limbal stem cell deficiency: contralateral versus ipsilateral autologous cultivated limbal epithelial transplantation. Am J Ophthalmol 2014;157(3):584-90 e1-2.

55. Rama P, Matuska S, Paganoni G, et al. Limbal stemcell therapy and long-term corneal regeneration. N Engl J Med 2010;363(2):147-55.

56. Vazirani J, Mariappan I, Ramamurthy S, et al. Surgical management of bilateral limbal stem cell deficiency. Ocul Surf 2016;14(3):350-64.

57. Baylis O, Figueiredo F, Henein C, Lako M, Ahmad S. 13 years of cultured limbal epithelial cell therapy: a review of the outcomes. J Cell Biochem 2011;112(4):993-1002.

58. Nishida K, Yamato M, Hayashida Y, et al. Corneal reconstruction with tissue-engineered cell sheets

J Dry Eye Ocul Sur Dis 2(1):e4-e11; June 10, 2019

This article is distributed under the terms of the Creative Commons Attribution-Non

Commercial 4.0 International License. (02019 Conto. 
composed of autologous oral mucosal epithelium. N Engl J Med 2004;351(12):1187-96.

59. Soma T, Hayashi R, Sugiyama H, et al. Maintenance and distribution of epithelial stem/progenitor cells after corneal reconstruction using oral mucosal epithelial cell sheets. PlosOne 2014;9(10):e110987.

60. Satake Y, Higa K, Tsubota K, Shimazaki J. Long-term outcome of cultivated oral mucosal epithelial sheet transplantation in treatment of total limbal stem cell deficiency. Ophthalmology 2011;118(8):1524-30.

61. Khan B, Dudenhoefer EJ, Dohlman CH. Keratoprosthesis: an update. Curr Opin Ophthalmol 2001;12(4):282-7.
62. Srikumaran D, Munoz B, Aldave AJ, et al. Long-term outcomes of boston type 1 keratoprosthesis implantation: a retrospective multicenter cohort. Ophthalmology 2014;121(11):2159-64.

63. Sareen D, Saghizadeh M, Ornelas L, et al. Differentiation of human limbal-derived induced pluripotent stem cells into limbal-like epithelium. Stem Cells Transl Med 2014;3(9):1002-12.

64. Nguyen MTB, Thakrar V, Chan CC. EyePrintPRO therapeutic scleral contact lens: indications and outcomes. Canadian J Ophthalmol J 2018;53(1):66-70. 